\title{
Sehr gute Sicht während der Behandlung
}

Gute Visualisierung im OP hat die chirurgische Praxis in den vergangenen Jahrzehnten stark verändert und die Behandlungsqualität sowie die -ergebnisse für Patienten enorm verbessert. In der Zahnheilkunde ist der Einsatz von Operationsmikroskopen - im Vergleich mit anderen Disziplinen wie der Neurochirurgie noch nicht überall Praxis. Seit nunmehr 15 Jahren profitieren jedoch immer mehr Zahnärzte von den Vorteilen innovativer Visualisierungslösungen von ZEISS für die Endodontie, die restorative Zahnheilkunde, die Implantologie und die Parodontologie. Auf der IDS präsentierte der Unternehmensbereich Medizintechnik von ZEISS sein erweitertes Portfolio für die Zahnheilkunde: Die digitale Kamera für das OPMI PROergo übermittelt Bilder aus dem Operationsmikroskop und unterstützt so Ärzte noch besser bei der Behandlung und im Patientengespräch; die LED-Beleuchtung EyeMag Light II für medizinische Kopflupen von ZEISS beleuchtet den Mundraum in Tageslichtqualität, sodass auch feine Details sichtbar werden. Darüber hinaus stellte ZEISS eine Publikation vor, in der Experten Schritt für Schritt und auf besonders anschauliche Weise die Vorteile der Mikroskopie in der Zahnheilkunde erläutern.

Nach einer Pressemitteilung der Carl Zeiss Meditec AG, Jena 